\title{
Map-Matching Using Shortest Paths
}

\author{
ERIN CHAMBERS, Saint Louis University \\ BRITTANY TERESE FASY, Montana State University \\ YUSU WANG, Ohio State University \\ CAROLA WENK, Tulane University
}

\begin{abstract}
We consider several variants of the map-matching problem, which seeks to find a path $Q$ in graph $G$ that has the smallest distance to a given trajectory $P$ (which is likely not to be exactly on the graph). In a typical application setting, $P$ models a noisy GPS trajectory from a person traveling on a road network, and the desired path $Q$ should ideally correspond to the actual path in $G$ that the person has traveled. Existing mapmatching algorithms in the literature consider all possible paths in $G$ as potential candidates for $Q$. We find solutions to the map-matching problem under different settings. In particular, we restrict the set of paths to shortest paths, or concatenations of shortest paths, in $G$. As a distance measure, we use the Fréchet distance, which is a suitable distance measure for curves since it takes the continuity of the curves into account.
\end{abstract}

CCS Concepts: • Theory of computation $\rightarrow$ Design and analysis of algorithms; Shortest paths; Theory and algorithms for application domains;

Additional Key Words and Phrases: Fréchet distance, graph matching, shortest paths

ACM Reference format:

Erin Chambers, Brittany Terese Fasy, Yusu Wang, and Carola Wenk. 2020. Map-Matching Using Shortest Paths. ACM Trans. Spatial Algorithms Syst. 6, 1, Article 6 (February 2020), 17 pages.

https://doi.org/10.1145/3368617

\section{INTRODUCTION}

The map-matching problem seeks to find a path $Q$ in a planar graph $G=(V, E)$ that has the smallest distance to $P$. In a typical application setting, $P$ models a noisy GPS trajectory from a person traveling on a road network, modeled as the planar graph $G$, and the desired path $Q$ should correspond to the actual path in $G$ that the person has traveled. Map-matching algorithms in the literature [1, 2,6] consider all possible paths in $G$ as potential candidates for $Q$ and apply similarity measures such as Hausdorff or Fréchet distance to compare input curves.

This work was generously support by the National Science Foundation under grants IIS-1319944, CCF-1054779, CCF1614562, DBI-1759807, CCF-1618605, CCF-1618247, and CCF-1618469.

Authors' addresses: E. Chambers, Department of Computer Science, Saint Louis University, 220 North Grand Avenue, St. Louis, MO 63103; email: echambe5@slu.edu; B. T. Fasy, School of Computing, Montana State University, 363 Barnard Hall, Bozeman, MT 59717; email: brittany@cs.montana.edu; Y. Wang, Computer Science and Engineering Department, Ohio State University, 2015 Neil Avenue, Columbus, OH 43210; email: yusu@cse.ohio-state.edu; C. Wenk, Department of Computer Science, Tulane University, 6823 St. Charles Avenue, New Orleans, LA 70118; email: cwenk@tulane.edu.

Permission to make digital or hard copies of all or part of this work for personal or classroom use is granted without fee provided that copies are not made or distributed for profit or commercial advantage and that copies bear this notice and the full citation on the first page. Copyrights for components of this work owned by others than ACM must be honored. Abstracting with credit is permitted. To copy otherwise, or republish, to post on servers or to redistribute to lists, requires prior specific permission and/or a fee. Request permissions from permissions@acm.org.

(C) 2020 Association for Computing Machinery.

2374-0353/2020/02-ART6 \$15.00

https://doi.org/10.1145/3368617

ACM Transactions on Spatial Algorithms and Systems, Vol. 6, No. 1, Article 6. Publication date: February 2020. 
We propose to restrict the set of potential paths in $G$ to a natural subset: those paths that correspond to shortest paths, or concatenations of shortest paths, in $G$. Restricting the set of paths to which a path can be matched makes sense in many settings. In particular, vehicles often follow routes computed by a navigation system, which often prefers certain types of routes over others. To the best of our knowledge, the current literature also does not consider the case where the vehicle makes multiple stops. For example, consider a person running several errands in one trip, where we are given the approximate path that the person followed, along with the underlying map. In this setting, knowledge of the number of stops and the type of path preferred (shortest travel time, shortest distance, or perhaps avoiding certain types of roads) can improve the final quality of the path that our algorithm matches to in the graph.

Related work. Map-matching is widely used in practice, such as to establish fast routes or points of interest from a large set of trajectories $[18,19]$. Common approaches include the use of Fréchet distance variants [2, 6], ad hoc incremental methods [6, Sec. 3], matching low-sampling-rate trajectories using spatial-temporal constraints [13], and hidden Markov models [15, 17]. Despite this, only few map-matching algorithms provide quality guarantees.

Only a small proportion of prior work considers restricting the set of paths in $G$. Instead, common practice reduces the space of paths by cropping $G$ inside an $\varepsilon$-neighborhood around $P$ before applying a general map-matching algorithm. Recently, Gheibi et al. [10] gave a map-matching algorithm that minimizes the sum of the lengths of walks on $P$ and $Q$ within some Fréchet distance. Their algorithm runs in $O(N m(N+m) \log (N+m))$ time and $O(N m(N+m))$ space, where $n=|V|, m=|E|, N=|P|$, and computes a shortest path in a discretized free space.

Our contribution. We provide algorithms for variants of the map-matching problem, in which the set of paths are restricted to shortest paths, or concatenations of shortest paths, in the graph. As a distance measure between paths, we use the Fréchet distance, which is a standard distance measure for curves in this setting that produces better matchings than other distance measures such as the Hausdorff distance, since it takes the continuity of the curves and not simply distance between them into account.

In Section 3, we provide an algorithm to match $P$ to the shortest possible path within some Fréchet distance in $G$. We prove properties of a distance function on the free space diagram, which is the main tool used to compute Fréchet distance. This allows us to use an incremental algorithm, which in turn uses less space than alternatives [10]. In Section 4, we give algorithms to match $P$ to concatenations of shortest paths in $G$ : in the min- $k$ variant, we find a path $Q$ in $G$ consisting of the smallest number $k$ of shortest path pieces that does not exceed a given Fréchet distance. In the min- $\varepsilon$ variant, we find a path $Q$ in $G$ consisting of at most $k$ shortest paths, for given $k$, such that the Fréchet distance to $P$ is minimized. We assume that breakpoints between shortest paths lie on vertices of $G$, and these breakpoints are mapped to vertices of $P$. In Section 5, we relax this constraint on the breakpoints and provide approximation algorithms that approximate the number of shortest path pieces and the Fréchet distance $\varepsilon$ when breakpoints can lie in the interior of edges in $G$ and can be mapped to the interior of edges of $P$.

To the best of our knowledge, we present the first systematic study of map-matching algorithms that consider a subset of paths with predefined properties in $G$ to be matched to $P$. Our article introduces a new perspective on map-matching and provides theoretical foundations for the practically relevant problem, where we consider a restricted set of path classes. We remark that an initial extended abstract of the present work appeared in the Third International Workshop on Interactive and Spatial Computing (IWISC) in 2018 [7]. The main difference lies in Section 5, which is a main technical component of the current work, where no technical proofs were provided for the earlier extended abstract. 


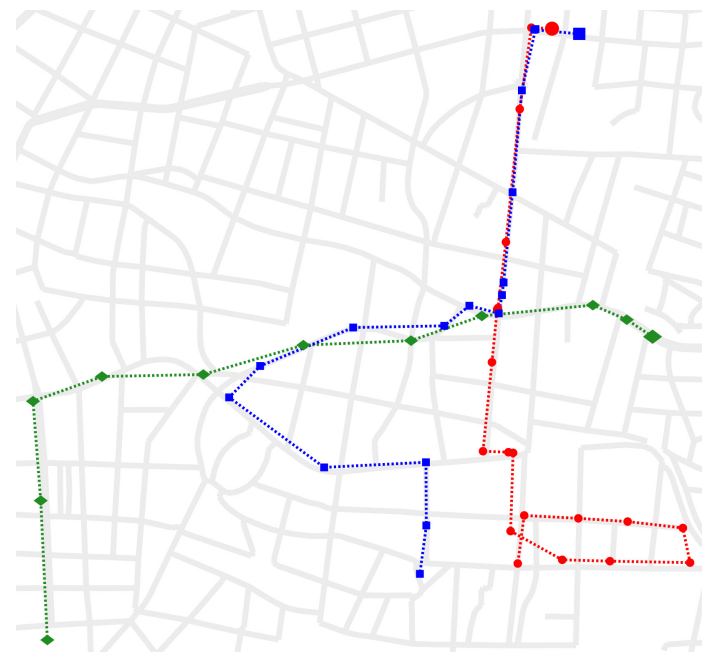

Fig. 1. Three school bus trajectories from the Athens-small dataset from mapconstruction.org.

Discussion of our model. Although it has been recognized that factors other than purely shortest distances may affect how people choose routes (e.g., see Zhu and Levinson [21]), many advanced models still rely heavily on the shortest path assumption (e.g., $[4,5,11,16,20])$.

Furthermore, we note that it has been observed before that shorter trajectories are more likely to be shortest paths, as do very long routes where the trajectory tends to follow a single route like a highway for most of the distance. For example, in Zhu and Levinson [21], the authors analyze GIS data from travel routes over an 8-week time frame, finding that nearly $34 \%$ of all trips follow the shortest time path exactly; when they relax to allow the route to be "nearly" shortest, then about $40 \%$ of all trips follow shortest routes. Routes also tend to revert to something close to a shortest path in the evening [14], as do trips associated with work as opposed to other tasks like shopping [8]. In another work [12], the authors analyze several datasets and show that in fact routes stay relatively close to the shortest path, within an ellipse whose two foci are the start and endpoints of the trajectory, and even within this area most deviations are small.

Although we measure the length of a path by its arc length using Euclidean distances in this article, we remark that the weight of a shortest path can be based on other quantities, such as travel times.

We also emphasize that assuming that a path consisting of $k$ shortest paths is fundamentally broader in scope than assuming that it is a single shortest path. Indeed, in the extreme case, by choosing a sufficiently large $k$, any path can fit into our model. For example, any path consisting of $n$ road segments can be considered as a concatenation of $k=n$ shortest paths.

In addition, several analyses suggest that breakpoints or anchors-key locations that attract a large number of trajectories-can be a natural way to decompose longer paths into routes that resemble optimal (or shortest) paths $[12,14]$. This helps to support investigating algorithms in a model where longer paths can be viewed as concatenations of shortest paths. For example, in Figure 1, bus route trajectories do not at all resemble shortest paths, but each can be broken into several subtrajectories that do follow a shortest route.

\section{PRELIMINARIES}

Let $G=(V, E)$ be a geometric graph with polygonal edges, and let $P$ be a polygonal path. We parameterize each (undirected) edge $e=(u, v) \in E$ linearly by $e(s):=(1-s) u+s v$ for $s \in[0,1]$, 


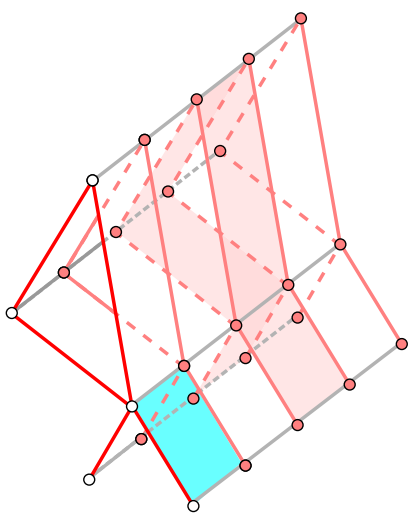

(a) Parameter space.

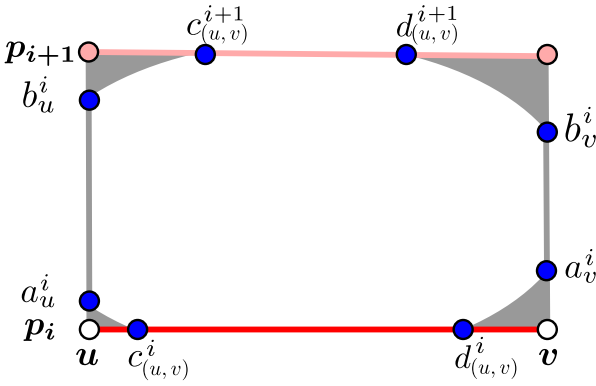

(b) A free space cell.

Fig. 2. On the left, we illustrate the parameter space $G \times P$, a graph $G$ (shown in red with white vertices) times a path $P$ of length four. For convenience, the path is drawn as a straight path. A slice is the graph cross an edge $e$ of the path: $G \times e$; see the shaded pink region. A level is the graph cross a vertex $v$ in the path: $G \times v$. Each level can be thought of as a copy of $G$. A cell corresponds to two edges, one of the path and one on $G$, as shown in cyan. On the right, we illustrate one free space of a cell $(u, v) \times\left(p_{i}, p_{i+1}\right)$, where $(u, v)$ is an edge in the graph and $p_{i}, p_{i+1}$ are consecutive points in $P$. The free space is equivalent to an ellipse intersecting this rectangle. Therefore, each edge of the rectangle has at most two points $(g, p)$ for which $\|g-p\|=\varepsilon$.

where the direction of the parameterization is fixed, but arbitrary. Let $p_{0}, p_{1}, \ldots, p_{N}$ be the sequence of $N+1$ vertices defining the polygonal path $P$. We identify each of these vertices with a point in the plane, and we parameterize each line segment edge $e_{i}=\left(p_{i}, p_{i+1}\right)$ linearly by $p_{i}(t):=(1-t) p_{i}+t p_{i+1}$ for $t \in[0,1]$. We use $P\left[p_{i}, p\right]$ to denote the polygonal subpath from $p_{i}$ to some other point $p \in G$.

The length of a path or subpath, either in $G$ or $P$, is simply the sum of all edge lengths in the path; in the case of partial edges, we use the fact that we have an arc length parameterization of all edges and take the arc length of the partial edge.

We are interested in finding a path in $G$ that is close to an input path $P$. To measure this closeness, we use the Fréchet distance [9]. Consider any two curves $\alpha, \beta:[0,1] \rightarrow R^{2}$. Let $\phi$ and $\psi$ be orientation-preserving homeomorphisms that serve as reparameterizations of $[0,1]$. We can measure the distance between $(\alpha \circ \phi)$ and $(\beta \circ \psi)$ pointwise and take the supremum. Then, the Fréchet distance $\delta_{F}(\alpha, \beta)$ is defined to be the infimum of this measurement over all reparameterizations $\phi$ and $\psi$. Formally: $\delta_{F}(\alpha, \beta)=\inf _{\phi, \psi} \sup _{s, t \in[0,1]}\|(\alpha \circ \phi)(t)-(\beta \circ \psi)(t)\|$. Intuitively, one can imagine a man walking along one curve and a dog along the other, continuously from beginning to end without backtracking. Then, the Fréchet distance is the shortest leash needed to connect the man and dog on their walk.

To match the path to the graph, we consider the cell complex $G \times P$ (Figure 2(a)). By convention, we say that the graph $G=(V, E)$ is horizontal and the path $P$ is vertical. For an edge $(u, v) \in E$ and consecutive path vertices $p_{i}$ and $p_{i+1}$, we consider the cell $(u, v) \times\left(p_{i}, p_{i+1}\right) \subseteq G \times P$ to be drawn with $(u, v)$ as a horizontal edge and $\left(p_{i}, p_{i+1}\right)$ as a vertical edge, as shown in Figure 2(b). A slice is the graph $G$ cross an edge $\left(p_{i}, p_{i+1}\right)$ of the path, $G \times\left(p_{i}, p_{i+1}\right)$, and a level is the graph cross a vertex $p_{i}$ of the path, $G \times p_{i}$.

For $\varepsilon>0$, the corresponding free space diagram $D_{\varepsilon}$ is the subset of $G \times P$ such that for all pairs $(g, p) \in D_{\varepsilon}$, the following inequality is satisfied: $\|g-p\| \leq \varepsilon$. The free space of a cell is equal to an ellipse intersected with the cell [3]. As a consequence, equality $\|g-p\|=\varepsilon$ holds for at most 
two points on each vertical or horizontal edge in the complex. On a vertical edge $u \times\left(p_{i}, p_{i+1}\right)$, we denote these two points by $a_{u}^{i}$ and $b_{u}^{i}$. Where appropriate, we slightly abuse notation and use $a_{u}^{i}$ to also identify the parameter $t$ for which $p_{i}(t)=a_{u}^{i}$. In this way, we say $a_{u}^{i} \leq b_{u}^{i}$. Likewise, on a horizontal edge $(u, v) \times p_{i}$, we denote $c_{u}^{i} \leq d_{u}^{i}$ as the points for which $\left\|c_{u}^{i}-p_{i}\right\|=\left\|d_{u}^{i}-p_{i}\right\|=\varepsilon$; Figure 2(b) presents an example of a labeled free space cell.

\section{SHORTEST AMONG MATCHING PATHS}

In this section, we consider only paths in $G$ that have restricted Fréchet distance to an input polygonal curve, and among those paths, we wish to find a shortest path. This means that we are interested in finding the shortest matching path.

Problem 1 (Shortest Matching Path). Given a parameter $\varepsilon>0$ and $a$ path $P$, find the shortest path in $G$ that is within Fréchet distance $\varepsilon$ to $P$.

We provide an incremental algorithm for computing such a shortest matching path. Our algorithm computes a distance function on all edges of the free space. We also prove properties of this distance functions that may be of independent interest.

\subsection{Algorithm}

Any path $Q$ in $G$ with $\delta_{F}(P, Q) \leq \varepsilon$ corresponds to a $P$-monotone path $\pi$ in free space $D_{\varepsilon} \subseteq G \times P$. A shortest such path $Q$ then corresponds to a shortest $P$-monotone path $\pi$ in $D_{\varepsilon}$, where the length of $\pi$ is only measured along $G$ (i.e., in the horizontal direction). Our algorithm follows a dynamic programming approach that combines the computation of paths in the free space diagram with shortest path computations.

We define a function $\varphi: G \times P \rightarrow \mathbb{R}$ such that $\varphi(g, p)=\min _{Q}|Q|$, where $Q$ ranges over all paths in $G$ ending at $g$ such that $\delta_{F}\left(P\left[p_{0}, p\right], Q\right) \leq \varepsilon$, and $|Q|$ denotes the length of $Q$. If no such path to $(g, p)$ exists, then $\varphi(g, p)=\infty$. In particular, $\varphi(g, p)=\infty$ for $(g, p) \notin D_{\varepsilon}$. We have that $\varphi(g, p)=$ $\min _{\pi}|\pi|$, where $\pi$ ranges over all $P$-monotone paths in $D_{\varepsilon}$ that end at $(g, p)$, and the length $|\pi|$ is measured along $G$ only. We call $\pi$ a $G$-shortest path, or shortest path for short. Thus, $\varphi$ captures the length of $G$-shortest paths in free space. Our algorithm computes $\varphi$ slice by slice over $G \times P$, with the goal to compute $\varphi\left(g, p_{N}\right)$ for some $g \in G$. Observe that a $G$-shortest path $\pi$ has to be monotone in each cell of $G \times P$. Therefore, it suffices to compute $\varphi$ on the vertical and horizontal edges of $G \times P$. In each slice of $G \times P$, we perform a Bellman-Ford inspired computation to propagate $\varphi$ between the vertical edges by relaxing along the horizontal edges.

For a vertical edge defined by $v \in V$ and an edge $\left(p_{i}, p_{i+1}\right)$ of the path, let $\varphi_{v, i}(t):[0,1] \rightarrow \mathbb{R}$ be defined by $\varphi_{v, i}(t)=\varphi\left(v, p_{i}(t)\right)$. For a horizontal edge defined by $e \in E$ and a vertex $p_{i}$ of the path, let $\varphi_{e, i}:[0,1] \rightarrow \mathbb{R}$ be defined by $\varphi_{e, i}(s):=\varphi\left(e(s), p_{i}\right)$. Note that for each (undirected) edge $(u, v) \in$ $E$, we only store one $\varphi$-function, say, $\varphi_{(u, v), i}(s)$, since $\varphi_{(v, u), i}(s)=\varphi_{(u, v), i}(1-s)$.

Lemma 3.1 (Vertical Monotonicity). The vertical function $\varphi_{v, i}(t)$ is monotone nonincreasing for $t \in\left[a_{v}^{i}, b_{v}^{i}\right]$.

Proof. Observe that $\left[a_{v}^{i}, b_{v}^{i}\right]$ corresponds to the intersection of the free space $D_{\varepsilon}$ with the vertical edge. Since $\varphi$ measures the length of paths in $D_{\varepsilon}$ in the $G$-direction only, paths can move in the vertical direction without increasing in length.

In particular, we note that a direct consequence of the preceding lemma is the fact that the minimum of this edge is attained at $a_{v}^{i}: \varphi_{v, i}\left(a_{v}^{i}\right) \leq \varphi_{v, i}(t)$ for all $t \in\left[a_{v}^{i}, b_{v}^{i}\right]$.

Our dynamic programming algorithm, Algorithm 1 , is based on the reachability propagation introduced by Alt and Godau [3] to compute the Fréchet distance. Instead of propagating binary 


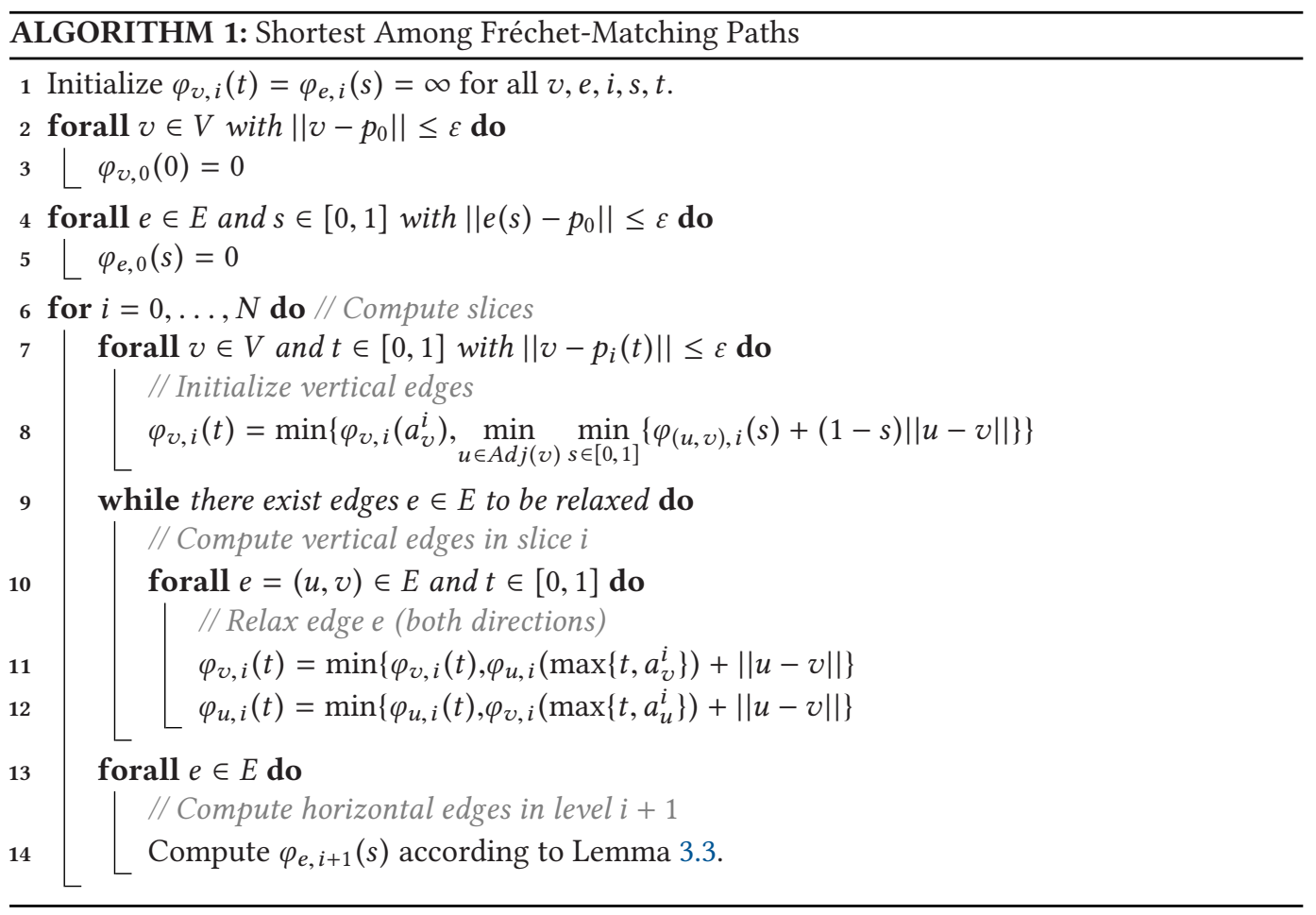

reachability information from cell to cell, we propagate function values for $\varphi$ along vertical and horizontal edges of $G \times P$. We will see in Lemma 3.4 and Lemma 3.5 that $\varphi$ is piecewise linear on a vertical or horizontal edge. We therefore store each $\varphi_{v, i}(t)$ and $\varphi_{e, i}(s)$ as a list of linear pieces. Updates such as the ones in lines 8,11, and 12 then take linear time in the length of the lists. The condition in line 9 is true if there exists an edge $e=(u, v)$ such that $\varphi_{v, i}(t)$ or $\varphi_{u, i}(t)$ are updated in lines 11 and 12 .

\subsection{Properties}

Algorithm 1 is based on the recursive formulas given in Lemma 3.2 and Lemma 3.3.

Lemma 3.2 (Compute Vertical Edges). Consider a vertical edge $v \times\left(p_{i}, p_{i+1}\right)$ for any $v \in V$ and $i \in\{0, \ldots, N\}$. Then, for any $t \in[0,1]$, we have the following:

- If $\left\|v-p_{i}(t)\right\|>\varepsilon$, then $\varphi_{v, i}(t)=\infty$.

- If $\left\|v-p_{i}(t)\right\| \leq \varepsilon$, then $\varphi_{v, 0}(0)=0$, and for $t \in(0,1]$ :

$$
\varphi_{v, i}(t)=\min \left\{\begin{array}{c}
\varphi_{v, i}\left(a_{v}^{i}\right), \\
\min _{u \in \operatorname{Adj}(v)} \varphi_{u, i}\left(\max \left\{t, a_{u}^{i}\right\}\right)+\|u-v\|, \\
\min _{u \in \operatorname{Adj}(v)} \min _{s \in[0,1]}\left\{\varphi_{(u, v), i}(s)+(1-s)\|u-v\|\right\}
\end{array}\right\} .
$$

Proof. The first two equalities follow directly from the definition of $\varphi$. To prove the third equality, consider a shortest monotone path $\pi$ in $D_{\varepsilon}$ ending at $\left(v, p_{i}(t)\right)$ for some $t \in[0,1]$. The last segment of $\pi$ connects to one of the following: 
(1) The bottom-most feasible point, $a_{v}^{i}$, on the same vertical edge $v \times\left(p_{i}, p_{i+1}\right)$,

(2) a point on a vertical edge $u \times\left(p_{i}, p_{i+1}\right)$ for a vertex $u \in V$ adjacent to $v$, or

(3) a point on a horizontal edge $(u, v) \times p_{i}$ for a vertex $u \in V$ adjacent to $v$.

A shortest monotone path always exists for which this last segment is a straight-line segment. The three cases correspond to the three values minimized over in the theorem. Measuring lengths in $G$, we observe that vertical paths in $D_{\varepsilon}$ have length zero. Hence, the length of the corresponding path in $G$ in the first case is $\varphi_{v, i}\left(a_{v}^{i}\right)$, the lengths in the other cases minimize over all vertices $u$ adjacent to $v$, and the value $\varphi_{v, i}(t)$ is the minimum of these three lengths. In the second case, the projection of $\pi$ onto $G$ traverses the entire edge $(u, v)$, which contributes length $\|u-v\|$. The third case minimizes over all possible connections to the horizontal edge $e \times p_{i}$ where $e=(u, v)$. A segment connecting $\left(v, p_{i}(t)\right)$ to a point $\left(e(s), p_{i}\right)$ has length $(1-s)\|u-v\|$, assuming $e$ is parameterized by $e(s)=(1-s) u+s v$.

Lemma 3.3 (Compute Horizontal Edges). Consider a horizontal edge $e \times p_{i+1}$ for any $e=$ $(u, v) \in E$ and $i \in\{0, \ldots, N\}$. Then, for any $\in[0,1]$ we have the following:

- $\varphi_{e, 0}(s)= \begin{cases}0, & \text { if }\left\|e(s)-p_{0}\right\| \leq \varepsilon \\ \infty, & \text { else }\end{cases}$

- If $\left\|e(s)-p_{i+1}\right\|>\varepsilon$, then $\varphi_{e, i+1}(s)=\infty$.

- If $\left\|e(s)-p_{i+1}\right\| \leq \varepsilon$, then

$$
\varphi_{e, i+1}(s)=\min \left\{\begin{array}{l}
\varphi_{u, i}\left(b_{u}^{i}\right)+s\|u-v\|, \\
\varphi_{v, i}\left(b_{v}^{i}\right)+(1-s)\|u-v\|, \\
\min _{s^{\prime} \in[0,1]}\left\{\varphi_{e, i}\left(s^{\prime}\right)+\left|s-s^{\prime}\right| \cdot\|u-v\|\right\}
\end{array}\right.
$$

Proof. The first two equalities follow directly from the definition of $\varphi$. It remains to prove the last equality given in Equation (1). Consider a shortest monotone path $\pi$ in $D_{\varepsilon}$ ending at $\left(e(s), p_{i+1}\right)$. The last segment of $\pi$ connects to one of the following:

(1) a point on the vertical edge $u \times\left(p_{i}, p_{i+1}\right)$,

(2) a point on the vertical edge $v \times\left(p_{i}, p_{i+1}\right)$, or

(3) a point on the horizontal edge $e \times p_{i}$.

These three cases correspond to the three values minimized over in Equation (1). By definition, $\varphi_{e, i+1}(s)$ is the minimum of these three values. In the first case, the last segment of $\pi$ connects to $b_{u}^{i}$ (or to a point below it on $u \times\left(p_{i}, p_{i+1}\right)$ with the same value of $\varphi$ ), since $\varphi_{v, i}(t)$ is monotone decreasing; the length of this segment is $s\|u-v\|$. The second case is analogous to the first case, for the other vertical edge in the free space cell. The third case minimizes over all possible connections to the horizontal edge $e \times p_{i}$. A segment connecting $\left(e(s), p_{i+1}\right)$ to a point $\left(e\left(s^{\prime}\right), p_{i}\right)$ has length $\left|s-s^{\prime}\right| \cdot\|u-v\|$.

The following two lemmas will be used to prove correctness of Algorithm 1 in Theorem 3.6.

Lemma 3.4 (Vertical Function Complexity). Let $v \times\left(p_{i}, p_{i+1}\right)$ be a vertical edge. Then, for $t \in$ $\left[a_{v}^{i}, b_{v}^{i}\right]$, the function $\varphi_{v, i}(t)$ is piecewise constant and monotone nonincreasing with complexity $O(n)$.

Proof. If $t \in\left[a_{v}^{1}, b_{v}^{i}\right]$, then $\phi_{v, i}(t) \leq \phi_{v, i}\left(a_{v}^{i}\right)$ since the path from $p\left(a_{v}^{1}\right)$ to $p(t)$ has length zero in $G$. The endpoints of each constant piece in $\varphi_{v, i}(t)$ can only be lower endpoints $a_{u}^{i}$ of the free space on vertical edges $u \times\left(p_{i}, p_{i+1}\right)$, for any $u \in V$. Hence, the complexity is $O(n)$.

Lemma 3.5 (Horizontal Function Complexity). Let $\times p_{i}$ be a horizontal edge. Then, the function $\varphi_{e, i}(s)$ is piecewise linear, for $s \in\left[c_{e}^{i}, d_{e}^{i}\right]$, where each piece is of slope $\|e\|,-\|e\|$ or zero. Note that such a function is necessarily $\|e\|$-Lipschitz. Furthermore, the complexity of $\varphi_{e, i}$ is $O(i)$. 
Proof. We prove this claim by induction on $i$. By definition, we know that $\varphi_{e, 0}(s)=0$ for all $s \in$ $\left[c_{e}^{0}, d_{e}^{0}\right]$. As a consequence of Lemma 3.3, we have that $\varphi_{e, i+1}$ is the lower envelope of a linear function with slope $\|e\|$, a linear function with slope $-\|e\|$, and $\min _{s^{\prime} \in[0,1]}\left\{\varphi_{e, i}\left(s^{\prime}\right)+\left|s-s^{\prime}\right| \cdot\|e\|\right\}$. Since, by inductive hypothesis, $\varphi_{e, i}$ is piecewise linear, where each piece is of slope $\|e\|,-\|e\|$ or zero, the minimum of the last term is attained as follows. If $s<c_{e}^{i}$ then $s^{\prime}=c_{e}^{i}$ and $c_{e}^{i} \leq s \leq d_{e}^{i}$, then $s^{\prime}=s$, and if $d_{e}^{i} \leq s$, then $s^{\prime}=d_{e}^{i}$. Hence, the function $\varphi_{e, i+1}$ consists of a translated copy of $\varphi_{e, i}$ with at most two additional linear pieces at each end. Therefore, we know that $\varphi_{e, i+1}$ has the desired structure, and its complexity is $O(i)$.

We prove the correctness and analyze the runtime of Algorithm 1 in the following theorem.

Theorem 3.6 (Correctness and Time Complexity). Algorithm 1 computes the length of a shortest matching path in $O(N(k m n+m N))$ time and $O\left(n^{2}+m N\right)$ space, where $k$ is the number of edges in the shortest matching path in $G$.

Proof. For each vertical edge $v \times\left(p_{i}, p_{i+1}\right)$ (and each horizontal edge $\left.e \times p_{i}\right)$, we compute $\varphi_{v, i}$ (and $\varphi_{e, i}$, respectively). The time for initialization (lines 1-5) is $O(n+m)$. From Lemma 3.4, we know that each $\varphi_{v, i}$ has complexity $O(n)$, and from Lemma 3.5, we know that each $\varphi_{e, i}$ has complexity $O(i)$. We use these discrete representations of $\varphi_{v, i}$ and $\varphi_{e, i}$ throughout the algorithm. Since the algorithm computes one slice at a time, we only need to store $\varphi_{v, i}$ and $\varphi_{e, i}$ for only one slice. Hence, the total storage complexity is $O\left(n^{2}+m N\right)$.

The correctness of the algorithm follows from Lemma 3.2 and Lemma 3.3. In particular, lines 7 through 12 are based on the recursive formula given in Lemma 3.2. All $\varphi_{v, i}$ on vertical edges $v \times\left(p_{i}, p_{i+1}\right)$ are initialized in lines 7 and 8 with values from the bottom horizontal edge. Then, lines 9 through 12 perform a Bellman-Ford shortest path propagation across all vertical edges in slice $i$. We continue the while loop in line 9 as long as at least one $\varphi_{v, i}\left(\right.$ or $\left.\varphi_{u, i}\right)$ was updated in lines 11 and 12. Hence, the number of iterations of the while loop is $k+1$ (once the shortest paths are found, no improvements will be made). After all, $\varphi_{v, i}$ have been computed in slice $i$, all $\varphi_{e, i+1}$ are computed from the vertical edges and the horizontal edges in level $i$, according to Lemma 3.3. Lines 7 and 8 take $O\left(n^{2}\right)$ time, lines 11 and 12 take $O(n)$ time, and line 14 takes $O(i)$ time. Hence, lines 6 through 14 of the algorithm take time $O\left(N\left(n^{2}+k m n+m N\right)\right)$, and thus the total runtime is $O(N(k m n+m N)$.

Remark 1. As stated, Algorithm 1 enforces monotonicity on $P$ but not on edges of $G=(V, E)$. If desired, the algorithm can be modified to enforce monotonicity on the edges in $E$ as follows. The cell complex would need to be defined using directed edges $E^{\prime}$, where undirected edges in $E$ are represented using two directed edges. The propagations according to Lemma 3.2 need to use adjacency lists $\operatorname{Adj}(v)=\left\{(u, v) \mid(u, v) \in E^{\prime}\right\}$. The horizontal propagation in Lemma 3.3 needs to be adjusted, by replacing Equation (1) with $\varphi_{e, i+1}(s)=\min \left\{\varphi_{u, i}\left(b_{u}^{i}\right)+s\|u-v\|, f_{e, i}(s)\right\}$. Here, $f_{e, i}(s)=0$ if $c_{e}^{i} \leq s \leq d_{e}^{i}$, and $f_{e, i}(s)=s-d_{e}^{i}$ if $d_{e}^{i}<s$. This formula models monotone propagation in the same way as in Alt and Godau [3], just that in addition to reachability, we propagate the length of a $G$-shortest path.

\section{MATCH TO CONCATENATION OF SHORTEST PATHS}

In this section, we are interested in matching the path $P$ to a concatenation of shortest paths in $G$. We consider two variants of the problem: one that minimizes the number of shortest paths that are concatenated and the other that minimizes the Fréchet distance $\delta_{F}$.

Problem $2($ Min- $k)$. Given a parameter $\varepsilon \geq 0$, find a path $Q$ in $G$ that is a concatenation of the smallest number of shortest paths in $G$ such that $\delta_{F}(P, Q) \leq \varepsilon$. 
Problem 3 (Min- $-\varepsilon$. Given a parameter $k \geq 1$, find a path $Q$ in $G$ that is a concatenation of at most $k$ shortest paths in $G$ such that the Fréchet distance between $P$ and $Q$ is minimized.

In this section, we assume that the paths in $G$ must begin and end at a vertex. We begin by exploring the case where $k=1$ in Section 4.1, then consider the more general case in Section 4.2 and Section 4.3. Allowing paths to start or end anywhere on an edge makes the problem considerably harder. We sketch approximation algorithms for this case in Section 5.

\subsection{Matching to Shortest Paths}

As a warm-up, we consider the min- $\varepsilon$ problem for the case where $k=1$-that is, we wish to find a shortest path $Q$ in $G$ that minimizes the Fréchet distance to $P$, among all shortest paths in $G$ that start and end at vertices in $V$.

First, we compute an implicit representation of all shortest paths between all pairs of vertices in $V$ by running Dijkstra's shortest path algorithm for each $s \in V$ as a source vertex. Shortest paths with a common start vertex are stored in a shortest path directed acyclic graph (DAG); note that although algorithms usually assume uniqueness of shortest paths and store only a tree, we wish to keep all possible shortest paths because we must store all of them to consider their Fréchet distance to $P$. The shortest path DAGs are computed and stored for each $s \in V$ as a source vertex, in total in $O(n(m+n \log n))$ time and $O\left(n^{2}\right)$ space.

Then, we need to compute the Fréchet distance between $P$ and each shortest path, to identify the minimum distance. We batch these computations by computing the Fréchet distance between a path and the entire shortest path DAGs. The following lemma and the resulting corollaries show that distances between shortest path prefixes and prefixes of $P$ can be computed efficiently in a batched manner. We state these results for a general DAG with a single root.

Lemma 4.1. Let $T=\left(V_{T}, E_{T}\right)$ be a DAG with a root $r$ and $\left|E_{T}\right|=m_{T}$. Let $P$ be a polygonal path with vertices $p_{0}, p_{1}, \ldots, p_{N}$. A path in $T$ from the root to a leaf that has the smallest Fréchet distance to $P$ can be computed in $O\left(m_{T} N \log \left(m_{T}+N\right)\right)$ time.

Proof. This is a simple modification of computation of the Fréchet distance for two polygonal paths by Alt and Godau [3] and a special case of the map-matching setting considered in Alt et al. [2]. For fixed $\varepsilon>0$, we compute the free space in $T \times P$. We then propagate reachability information from $\left(r, p_{0}\right)$ in dynamic programming fashion in this free space. Starting with filling reachability information in $r \times P$, we then propagate the reachability monotonically across both $T$ and $P$, traversing $T$ in an order determined by a topological sort of $T$, and $P$ from $p_{0}$ to $p_{N}$. For each edge $(u, v) \in E_{T}$, the reachable points in $(u, v) \times P$ are computed by straightforward propagation from the reachable points in $u \times P$. But since $v$ may have multiple incoming edges, the reachability information for $v \times P$ is then computed as the union of all propagated reachability information for all $(u, v) \in E_{T}$. It takes time and space $O\left(m_{T} N\right)$ to solve the decision problem. With parametric search $[2,3]$, the path in $T$ from the root to a leaf that has the smallest Fréchet distance to $P$ can be found in $O\left(m_{T} N \log \left(m_{T}+N\right)\right)$ time.

For fixed $\varepsilon>0$, the algorithm described in the proof of Lemma 4.1 does in fact compute reachability information for all paths starting in the root of $T$ and all prefixes of $P$.

Corollary 4.2. Let $T=\left(V_{T}, E_{T}\right)$ be a DAG with a rootr and $\left|E_{T}\right|=m_{T}$, and let $\varepsilon>0 . \operatorname{In} O\left(m_{T} N\right)$ time, one can compute for all points $g \in T$ and $p \in P$ whether there exists a path $Q_{r, g}$ in $G$ from $r$ to $g$ such that $\delta_{F}\left(Q_{r, g}, P\left[p_{0}, p\right]\right) \leq \varepsilon$.

And in fact, reachability can be computed efficiently if either the start point of the path $P$ or the start point of a corresponding path in $T$ is allowed to vary along an edge. 
Corollary 4.3. Let $T=\left(V_{T}, E_{T}\right)$ be a DAG with root $r$, and let $\left|E_{T}\right|=m_{T}$ and $\varepsilon>0$. The following can be computed in $O\left(m_{T} N\right)$ time:

(i) For all points $g \in T, p \in P$, and $x \in\left(p_{0}, p_{1}\right)$ whether there exists a path $Q_{r, g}$ in $G$ from $r$ to $g$ such that $\delta_{F}\left(Q_{r, g}, P[x, p]\right) \leq \varepsilon$.

(ii) If $(r, v)$ is the only edge incident on the root, then it can be computed for all points $g \in T, p \in P$, and $x \in(r, v)$ whether there exists a path $Q_{x, g}$ in $G$ from $x$ tog such that $\delta_{F}\left(Q_{x, g}, P\left[p_{0}, p\right]\right) \leq \varepsilon$.

Proof. For (i), a simple modification of the reachability initialization step in the proof of Lemma 4.1 results in computing reachability from $(r, x)$ for any $x \in\left(p_{0}, p_{1}\right)$. For (ii), if $g \notin(r, v)$, then a simple modification of the reachability initialization step in the proof of Lemma 4.1 results in computing reachability from any $x \in(r, v)$. If both $x$ and $g$ are on the same edge $(r, v)$, then we compute the reachability in $(r, v) \times P$ directly.

We apply Lemma 4.1 to the shortest path DAG $T_{s}$ for each start vertex $s \in V$. We compute a shortest path in $T_{s}$ that has the smallest Fréchet distance to $P$ in $O(m N \log (m+N))$ time. Repeating this for each source vertex, and accounting for running Dijkstra's algorithm in the beginning, results in a total runtime of $O(n m N \log (m+N))$ and $O(n(n+N))$ space. We summarize our result in the following theorem.

Theorem 4.4 (Matching to Shortest Path). A path $Q$ that minimizes the Fréchet distance to $P$, among all shortest paths in $G$ that start and end at vertices in $V$, can be computed in $O(n m N \log (m+$ $N))$ and $O(n(n+N))$ space.

\subsection{The Min- $k$ Problem}

In this section, we solve the min- $k$ problem. For fixed $\varepsilon \geq 0$, we wish to find a path $Q$ that is a concatenation of the smallest number of shortest paths in $G$ such that $\delta_{F}(P, Q) \leq \varepsilon$. We require that all shortest paths start and end at vertices in $V$.

Auxiliary graph. We build an auxiliary graph $G^{\prime}=\left(V^{\prime}, E^{\prime}\right)$ as follows. The set of vertices $V^{\prime}$ are ordered pairs of a vertex in $V$ and a vertex in $P$; formally, we write: $V^{\prime}=\left\{\left\langle v, p_{i}\right\rangle \mid v \in V, i \in\right.$ $\{0, \ldots, N\}\}$. There is an edge in $E^{\prime}$ connecting $\left\langle u, p_{i}\right\rangle$ and $\left\langle v, p_{j}\right\rangle$, if there is a shortest path $Q$ in $G$ from $u$ to $v$ such that the Fréchet distance between $P[i, j]$ and $Q$ is at most $\varepsilon$. Formally, we have $E^{\prime}=\left\{\left(\left\langle u, p_{i}\right\rangle,\left\langle v, p_{j}\right\rangle\right) \mid 0 \leq i \leq j \leq N\right.$, and there is a shortest path $Q$ from $u$ to $v$ in $G$ such that $\left.\delta_{F}(Q, P[i, j]) \leq \varepsilon\right\}$. We have $\left|V^{\prime}\right|=n N$ and $\left|E^{\prime}\right| \in O\left(n^{2} N^{2}\right)$.

This auxiliary graph can be constructed as follows. We compute all shortest path DAGs $T_{u}$ by running Dijkstra's shortest path algorithm for every $u \in V$. For fixed $u \in V$ and $i \in\{0 \leq i \leq N\}$, we use Corollary 4.2 to compute the reachability information. For each $v \in V$ and $i \leq j \leq N$, we can then read off whether there exists a shortest path in $G$ from $u$ to $v$ such that $\delta_{F}\left(Q_{u, v}, P[i, j]\right) \leq \varepsilon$. This determines whether $\left(\left\langle u, p_{i}\right\rangle,\left\langle v, p_{j}\right\rangle\right) \in E^{\prime}$. The runtime is $O(n(m+n \log n))$ to compute all shortest path DAGs, $O(m N)$ to compute the edges for fixed $u$ and $i$, and hence $O\left(n\left(m N^{2}+n \log n\right)\right)$ time total to compute $E^{\prime}$.

Algorithm. We can now solve our problem by finding a shortest path in $G^{\prime}$, starting at any vertex $\left\langle u, p_{0}\right\rangle$ for any $u \in V$ and ending at any vertex $\left\langle v, p_{N}\right\rangle$. We connect a supersource $\hat{s}$ to all $\left\langle u, p_{0}\right\rangle$ for any $u \in V$. Since the length of the path is determined by the number of edges, we can compute such shortest paths by running breadth-first search from $\hat{s}$ in time $O\left(\left|V^{\prime}\right|+\left|E^{\prime}\right|\right)=O\left(n^{2} N^{2}\right)$. The total runtime is dominated by the time $O\left(n\left(m N^{2}+n \log n\right)\right)$ to compute the auxiliary graph. We summarize our result in the following theorem. 
Theorem 4.5 (Min- $k$ ). For fixed $\varepsilon \geq 0$, a path $Q$ that is a concatenation of the smallest number of shortest paths in $G$ such that $\delta_{F}(P, Q) \leq \varepsilon$ can be computed in $O\left(n\left(m N^{2}+n \log n\right)\right)$ time and $O\left(n^{2} N^{2}\right)$ space.

\subsection{Min- $\varepsilon$}

Next, we show how we can use our solution for the min- $k$ problem described in Section 4.2 to develop a solution for the min- $\varepsilon$ problem. For fixed $k \geq 2$, we wish to find a path $Q$ that is a concatenation of at most $k$ shortest paths in $G$ such that $\delta_{F}(P, Q)$ is minimized. Again, we require that all shortest paths start and end at vertices in $V$.

Let $k \geq 2$ be fixed. We modify the algorithm described in Section 4.2 to serve as a decision procedure for a given $\varepsilon \geq 0$ : return true if a shortest path exists of length $\leq k$ and false otherwise. We optimize $\varepsilon$ by performing a binary search on a superset of the critical values for $\varepsilon$, which are values for which solutions to the decision procedure changes combinatorially. These changes are caused by combinatorial changes in the free space diagram for a shortest path $Q$ and $P$; see Alt and Godau [3]. We consider all possible critical values within each free space cell and across pairs of free space cells. Possible critical values are those $\varepsilon$ for which $a_{u}^{i}=b_{v}^{j}$ or $c_{e}^{i}=d_{e}^{j}$ for $u, v \in V, e \in$ $E$, and $j=i$ or $j=i+1$. There are $O\left(n^{2} N+N^{2} n\right)$ such values that constitute a superset of the combinatorial changes that affect our decision procedure. We sort these critical values in $O\left(\left(n^{2} N+\right.\right.$ $\left.\left.N^{2} n\right) \log (n+N)\right)$ time and perform a binary search using the decision procedure, which results in a total runtime of $O\left(n\left(m N^{2}+n \log n\right) \log (n+N)\right)$. We summarize our result as follows.

Theorem $4.6($ Min- $\varepsilon)$. For fixed $k \geq 0$, a path $Q$ that is a concatenation of at most $k$ shortest paths in $G$ such that $\delta_{F}(P, Q)$ is minimized, can be computed in $O\left(n\left(m N^{2}+n \log n\right) \log (n+N)\right)$ time and $O\left(n^{2} N^{2}\right)$ space.

\section{APPROXIMATION ALGORITHM FOR $k$-SP WITHOUT VERTEX CONSTRAINT}

In this section, we consider the more general version of the $k$-shortest path problem by removing the vertex constraint. Let $|G|$ denote the underlying space of $G$, comprising all points in $G$, including those in the interior of edges. We say that a path $Q \subset|G|$ is a $k-S P$ if it can be partitioned into $k$ consecutive pieces $Q=Q_{1} \circ Q_{2} \cdots \circ Q_{k}$ such that each $Q_{i}$ is a shortest path between its two endpoints in $|G|$. Let $P=\left\{P_{1}, \ldots, P_{k}\right\}$ be a $k$-partitioning of the underlying space $|P|$ of the polygonal curve $P$-that is, $|P|=P_{1} \circ P_{2} \cdots \circ P_{k}$ with $P_{i}$ and $P_{j}$ disjoint in their interior for all $i \neq j$. We say that $G$ has a $(k, \varepsilon)$-matching for $P$ if there exists a $k$-SP $Q=Q_{1} \circ Q_{2} \cdots \circ Q_{k}$ and a $k$-partitioning $P=$ $\left\{P_{1}, P_{2}, \ldots P_{k}\right\}$ such that for any $i \in[1, k]$, the Fréchet distance is bounded: $\delta_{F}\left(Q_{i}, P_{i}\right) \leq \varepsilon$. (This also implies that $\delta_{F}(Q, P) \leq \varepsilon$.) We refer to endpoints of each path in $Q$ and in $P$ as breakpoints. Note that the breakpoints could lie in the interior of edges.

Problem 4. Given $k$ and $\varepsilon>0$, the goal is to decide whether there exists a $k-S P Q=Q_{1} \circ Q_{2} \cdots \circ$ $Q_{k}$ and a $k$-partitioning $P=\left\{P_{1}, P_{2}, \ldots P_{k}\right\}$ of $P$ such that for any $i \in[1, k]$, the Fréchet distance is bounded: $\delta_{F}\left(Q_{i}, P_{i}\right) \leq \varepsilon$.

This general version of the problem seems to be much more challenging. For example, consider Figure 3. Suppose we already know that point $p_{0}$ should be matched to some point on edge $e_{1}=$ $\left(u_{1}, u_{2}\right)$, and the last point $p_{N}$ should be matched to some point on edge $e_{2}=\left(w_{1}, w_{2}\right)$. Let $\pi_{1}$ be a shortest path from $u_{1}$ to $w_{1}$, and let $\pi_{2}$ be a shortest path from $u_{2}$ to $w_{2}$. We need to compute a shortest path starting in some $u \in e_{1}$ and ending in some $w \in e_{2}$ whose Fréchet distance to $P$ is at most $\varepsilon$. However, whether the path $u \leadsto \pi_{1} \leadsto w$ or the path $u \leadsto \pi_{2} \leadsto w$ is shortest depends on the positions of both $u$ and $w$. Hence, the endpoint $w$ depends on the starting point $u$, which makes developing a dynamic programming strategy challenging. 


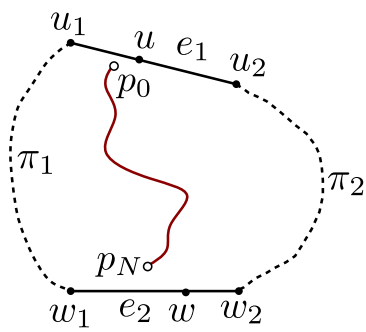

Fig. 3. The path $u \leadsto \pi_{1} \leadsto w$ or the path $u \leadsto \pi_{2} \leadsto w$ may be shortest, depending on the positions of $u$ and $w$, where $w$ depends on $u$.

In this section, we focus on approximation algorithms. We say that an algorithm is an $(\alpha, \beta)$ approximation for the $(k, \varepsilon)$-matching problem if it computes an $(\alpha k, \beta \varepsilon)$-matching for the path $P$ whenever there exists a $(k, \varepsilon)$-matching for $P$ in $G$. In what follows, we describe such an approximation algorithm, where the input satisfies the following mild assumption:

Assumption-R: For the optimal $k$-SP $Q$, there is no U-turn in the interior of an edge. Equivalently, for a breakpoint $s_{i}$ connecting shortest path pieces $Q_{i}$ and $Q_{i+1}$, if $s_{i}$ is in the interior of edge $e=(u, v)$, then $Q_{i} \cap Q_{i+1} \cap e=\left\{s_{i}\right\}$.

Remark. From a technical point of view, this assumption is important in proving Proposition 5.2. It may be possible to remove this assumption and still achieve a $G$-restricted $(2 k, \varepsilon)$, as in Proposition 5.2 with a more complicated argument. However, we note that this assumption is in fact natural, or even necessary for a real route planning. Indeed, assuming each edge in the graph represents a road segment connecting two junction nodes, many jurisdictions do not allowed a U-turn between the junction nodes.

Theorem 5.1 (Approximation Theorem). Letting $P$ be a polygonal path and $G=(V, E)$ be a graph satisfying Assumption- $\mathrm{R}$, there is a $(2,2)$-approximation algorithm for the $(k, \varepsilon)$-matching problem with running time $O\left(n m N^{2}\right)$, where $n=|V|, m=|E|$, and $N=|P|$.

To prove Theorem 5.1, we solve a version of the $k$-matching problem for which we require that all breakpoints in the $k$-SP $Q$, other than the start point and endpoint, have to be vertices from the graph $G$. We call this the $G$-restricted $(k, \varepsilon)$-matching problem for $P$. Theorem 5.1 follows immediately from the following two propositions.

Proposition 5.2. If there is a $(k, \varepsilon)$-matching between $P$ and $G$, where the input satisfies Assumption- $\mathrm{R}$, then there is a G-restricted $(2 k, \varepsilon)$-matching between $P$ and $G$.

Proposition 5.3. Given a polygonal path $P$ and a graph $G=(V, E)$, there is a $(1,2)$ approximation algorithm for the $G$-restricted $(k, \varepsilon)$-matching problem whose running time is $O\left(n m N^{2}\right)$, where $n=|V|, m=|E|$, and $N=|P|$.

Proof of Proposition 5.2. Assume $G$ has a $(k, \varepsilon)$-matching, and let $Q^{*}=Q_{1} \circ Q_{2} \circ \cdots \circ Q_{k}$ be the $k$-SP and $P=\left\{P_{1}, P_{2}, \ldots, P_{k}\right\}$ be the $k$-partition in this matching. We now show that we can modify $Q$ to a $G$-restricted $2 k$-SP $\widehat{Q}$ forming a $(2 k, \varepsilon)$-matching with some $2 k$-partition of $P$. Our modification re-partitions $P$ and $Q^{*}$. Note that for any oriented path $\pi$, given an ordered sequence of points $\left\{\alpha_{0}, \ldots, \alpha_{\ell}\right\}$ along this path with start point $\alpha_{0}$ and endpoint $\alpha_{\ell}$, it induces a unique partition $\pi\left[\alpha_{0}, \alpha_{1}\right] \circ \pi\left[\alpha_{1}, \alpha_{2}\right] \circ \cdots \circ \pi\left[\alpha_{\ell-1}, \alpha_{\ell}\right]$ of $\pi$. (Recall that $\pi[\alpha, \beta]$ is the subcurve of $\pi$ between two points $\alpha \leq \beta$, meaning that $\alpha$ has a smaller preimage than $\beta$ under the parameterization of $\pi$.) Hence, in what follows, we simply specify such sequences of breakpoints to describe (re)partitioning of the paths $Q^{*}$ and $P$. 


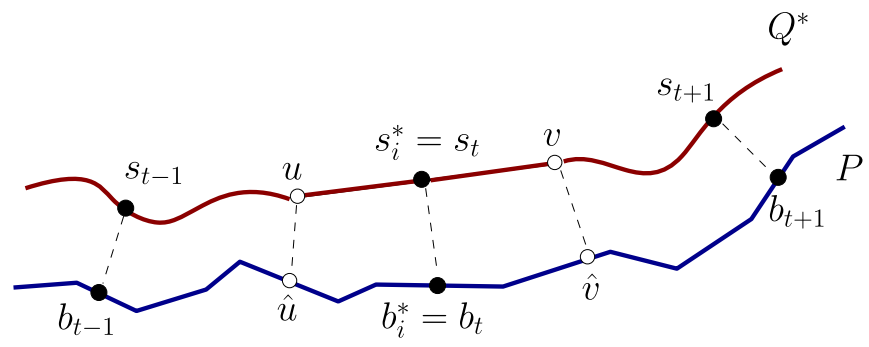

Fig. 4. Black dots are breakpoints, and dashed segments indicate aligned points.

Definition 5.4. Repartitions of $Q^{*}$ and $P$ induced by a sequence of breakpoints $S=\left\{s_{0}, \ldots, s_{\ell}\right\}$ and $\Pi=\left\{b_{0}, \ldots, b_{\ell}\right\}$ are called valid if for all $i \in[0, \ell-1]$,

(i) each piece $Q^{*}\left[s_{i}, s_{i+1}\right]$ is a shortest path in $G$, and

(ii) $\delta_{F}\left(Q^{*}\left[s_{i}, s_{i+1}\right], P\left[b_{i}, b_{i+1}\right]\right) \leq \varepsilon$.

Let $S^{*}=\left\{s_{0}^{*}, s_{1}^{*}, \ldots, s_{k}^{*}\right\}$ be the sequence of breakpoints of the optimal $k$-SP $Q^{*}$, and let $\Pi^{*}=$ $\left\{b_{0}=p_{0}^{*}, b_{1}^{*}, \ldots, b_{k-1}^{*}, b_{k}^{*}=p_{n_{p}}\right\}$ be the sequence of breakpoints for the optimal $k$-partition of $P$. We now process each $s_{i}^{*}$ in order from $i=1$ to $i=k-1$. In the beginning, $S_{0}=S^{*}$ and $\Pi_{0}=P^{*}$. In the $i$-th iteration, we obtain $S_{i}$ from $S_{i-1}$ such that $\left|S_{i}\right| \leq\left|S_{i-1}\right|+1$, and we obtain $\Pi_{i}$ from $\Pi_{i-1}$ such that $\left|\Pi_{i}\right| \leq\left|\Pi_{i-1}\right|+1$. We also maintain the invariant that the partitions $S_{i}$ and $\Pi_{i}$ are valid.

Specifically, in the $i$-th iteration, suppose the breakpoint $s_{i}^{*}$ from the optimal $k$-SP is still present in $S_{i-1}$. Assume $s_{i}^{*} \in e=(u, v) \in E$. By Assumption-R, the entire edge $(u, v)$ must be covered by the path $Q^{*}$ and $Q^{*}[u, v]=(u, v)$. Let $S_{i-1}=\left\{s_{0}, \ldots, s_{\ell}\right\}$ and $\Pi_{i-1}=\left\{b_{0}, \ldots, b_{\ell}\right\}$. Since $S_{i-1}$ and $\Pi_{i-1}$ are valid, there exist Fréchet matching $F$ between $Q^{*}$ and $P$ composed by the union of Fréchet matchings between $Q^{*}\left[s_{j}, s_{j+1}\right]$ and $P\left[b_{j}, b_{j+1}\right]$ for all $j \in[1, \ell)$ such that $\delta_{F}\left(Q^{*}\left[s_{j}, s_{j+1}\right], P\left[b_{j}, b_{j+1}\right]\right) \leq \varepsilon$. Let $\hat{u}$ and $\hat{v}$ be two points aligned to $u$ and $v$ under this matching $F$; obviously, $\delta_{F}((u, v), P(\hat{u}, \hat{v})) \leq \varepsilon$. See Figure 4 for an illustration, where suppose $s_{i}^{*}=s_{i} \in S_{i-1}$.

We obtain $S_{i}$ by removing $s_{i}^{*}$ as a breakpoint from $S_{i-1}$ and adding $u$ and $v$ as new breakpoints to $S_{i-1}$. (In general, the edge $(u, v)$ may contain more breakpoints than $s_{i}^{*}$, and we need to remove all of them from $S_{i-1}$.) Similarly, we add $\hat{u}$ and $\hat{v}$ as new breakpoints and remove any existing breakpoints of $\Pi_{i-1}$ contained in $P[\hat{u}, \hat{v}]$ (the breakpoint $b_{i}$ that $s_{i}$ is matched to will necessarily be removed). This gives rise to a pair of new partitions $S_{i}$ and $\Pi_{i}$, where the number of pieces can increment by at most one.

We now argue that $S_{i}$ and $\Pi_{i}$ are also valid. We already know that $S_{i-1}=\left\{s_{0}, \ldots, s_{\ell}\right\}$ and $\Pi_{i-1}=$ $\left\{b_{0}, \ldots, b_{\ell}\right\}$ are valid. Consider any two consecutive breakpoints in $S_{i}$. Then, one of the following three cases must hold:

(1) Both breakpoints $s_{j}, s_{j+1} \in S_{i-1} \cap S_{i}$. Then, as $S_{i-1}$ and $\Pi_{i-1}$ are valid, we have that the two conditions (i) and (ii) in Definition 5.4 hold for $s_{j}$ and $s_{j+1}$.

(2) We have a pair of new consecutive breakpoints $u$ and $v$ in $S_{i}$, corresponding to new breakpoints $\hat{u}$ and $\hat{v}$ in $\Pi_{i-1}$. However, we know that $Q[u, v]=(u, v)$, and thus it is a shortest path between $u$ and $v$; and also we know from earlier that $\delta_{F}((u, v), P(\hat{u}, \hat{v})) \leq \varepsilon$.

(3) The two consecutive breakpoints in $S_{i}$ are either of the form $s_{j}, u(j=t-1$ in Figure 4) or, symmetrically, are $v, s_{j^{\prime}}$ from $S_{i}\left(j^{\prime}=t+1\right.$ in Figure 4). Consider $s_{j}$ and $u$ from $S_{i}$, which correspond to consecutive breakpoints $b_{j}, \hat{u}$ from $\Pi_{i}$. By construction, $Q^{*}\left[s_{j}, u\right] \subseteq Q^{*}\left[s_{j}, s_{j+1}\right]$; thus, $Q^{*}\left[s_{j}, u\right]$ is necessarily a shortest path of $G$ as well. Furthermore, $P\left[b_{j}, \hat{u}\right] \subseteq P\left[b_{j}, b_{j+1}\right]$ and $u$ is aligned to $\hat{u}$ under the Fréchet matching $F$ between 
$Q^{*}\left[s_{j}, s_{j+1}\right]$ and $P\left[b_{j}, b_{j+1}\right]$ mentioned earlier. Thus, we have $\delta_{F}\left(Q^{*}\left[s_{j}, u\right], P\left[b_{j}, \hat{u}\right]\right) \leq \varepsilon$ under the same matching $F$. Symmetrically, we can argue that $\delta_{F}\left(Q^{*}\left[v, s_{j^{\prime}}\right], P\left[\hat{v}, b_{j^{\prime}}\right]\right) \leq \varepsilon$.

It then follows that $S_{i}$ and $\Pi_{i}$ are valid. Furthermore, after each iteration, if breakpoint $s_{i}^{*}$ is in the interior of an edge in $G$, then we remove at least this breakpoint and add two new breakpoints $u$ and $v$, which are vertices of graph $G$. Thus, in each iteration, the number of breakpoints can increase by at most one, implying that $\left|S_{k}\right| \leq 2 k+1$. After processing all $s_{i}^{*}$ 's, all breakpoints from $Q^{*}$ that are in the interior of graph edges are removed, and all newly added breakpoints are graph nodes. Hence, the new partitions $S_{k}$ of $Q^{*}$ and $\Pi_{k}$ of $P$ witness a $G$-restricted $(2 k, \varepsilon)$-matching for $P$ in $G$, which proves Proposition 5.2.

Proof of Proposition 5.3. We now describe a $(1,2)$-approximation algorithm for the $G$ restricted case, which would then prove Proposition 5.3. To make the main idea clear, we first assume that in the $G$-restricted $(k, \varepsilon)$-matching, all breakpoints of the $k$-SP $Q$ have to be vertices in $G$ (in our earlier definition, the start point and endpoint may not be). At the end of this proof, we will describe how to remove this assumption.

The high-level framework is similar to the approach in Section 4.2. Given parameter $\varepsilon$, we build an auxiliary graph $G^{\prime}=\left(V^{\prime}, E^{\prime}\right)$ as follows. The node set $V^{\prime}$ consists of $\left\{\left\langle v, e_{i}\right\rangle \mid v \in V, e_{i}=\right.$ $\left(p_{i}, p_{i+1}\right)$ is the $i$-th edge in $\left.P\right\}$. The edge $\left(\left\langle v, e_{i}\right\rangle,\left\langle v^{\prime}, e_{j}\right\rangle\right) \in E^{\prime}$ is in the auxiliary graph $G^{\prime}$ if and only if there is a subpath $P[a, b] \subseteq P$ with $a \in e_{i}$ and $b \in e_{j}$, as well as a shortest path $Q$ from $v \in V$ to $v^{\prime} \in V$ in $G$, such that $\delta_{F}(Q, P[a, b]) \leq \varepsilon$; if $i=0$, then we require $a=p_{0}$, and if $j=N-1$, then we require $b=p_{N}$. The latter two conditions are to guarantee that the first and last breakpoints for the partition of $P$ have to be $p_{0}$ and $p_{N}$, respectively. Our algorithm returns "yes" if a path of at most $k$ links exists from $\left\langle s, e_{0}\right\rangle$ to $\left\langle t, e_{N}\right\rangle$ in the auxiliary graph $G^{\prime}$ for some $s, t \in V$.

We compute the edge set $E^{\prime}$ for this auxiliary graph as follows. We add the edge $\left(\left\langle v, e_{i}\right\rangle,\left\langle v^{\prime}, e_{j}\right\rangle\right)$ to the auxiliary graph $G^{\prime}$ as long as there is a monotone path in the free space $D_{v}$ starting from some point, say $(v, a)$, along the vertical edge $v \times e_{i}$, to some point, say $(v, b)$, within the vertical edge $v^{\prime} \times e_{j}$. We further associate the pair $(a, b)$ with the edge $\left(\left\langle v, e_{i}\right\rangle,\left\langle v^{\prime}, e_{j}\right\rangle\right) \in E^{\prime}$, and we say that $(a, b)$, with $a \in e_{i}$ and $b \in e_{j}$, witnesses the existence of the edge $\left(\left\langle v, e_{i}\right\rangle,\left\langle v^{\prime}, e_{j}\right\rangle\right)$. Using Corollary 4.3 for the shortest path DAG $T_{v}$ for $v \in V$, this can be computed in $O\left(\left|T_{v}\right| N\right)=O(m N)$ time for fixed $v$ and $i$.

Overall, this auxiliary graph has $\left|V^{\prime}\right|=O(n N)$ nodes and $\left|E^{\prime}\right|=O\left(n^{2} N^{2}\right)$ edges. Constructing all edges takes $O\left(\left|V^{\prime}\right| m N\right)=O\left(n m N^{2}\right)$ total time. We need to test whether there is a path from $\left\langle s, e_{0}\right\rangle$ to $\left\langle t, e_{N}\right\rangle$ of at most $k$ links in $G^{\prime}$ for some $s, t \in V$. This can be done in $O\left(\left|V^{\prime}\right|+\left|E^{\prime}\right|\right)=O\left(n m N^{2}\right)$ time by adding a supersource node as in the algorithm for Theorem 4.5. Hence, the claimed time complexity in Proposition 5.3 follows.

To prove the correctness of our algorithm, we will show how to construct a $G$-restricted $(k, 2 \varepsilon)$ matching from the $k$-link path mentioned earlier from the auxiliary graph. Let

$$
\begin{aligned}
u_{0} & =\left\langle s=s_{0}, p_{0}\right\rangle, u_{1}=\left\langle s_{1}, e_{i_{1}}\right\rangle, \ldots, \\
u_{k-1} & =\left\langle s_{k-1}, e_{i_{k-1}}\right\rangle, u_{k}=\left\langle s_{k}=t, p_{N}\right\rangle
\end{aligned}
$$

be the sequence of nodes for a path of $k$ links from $u_{0}=\left\langle s, p_{0}\right\rangle$ to $u_{k}=\left\langle t, p_{N-1}\right\rangle$ in the auxiliary graph $G^{\prime}$. Obviously, this gives rise to a $k$-SP $Q=Q_{1} \circ Q_{2} \circ \cdots Q_{k}$, where for each $j \in[0, k-1], Q_{j}$ is a shortest path from $s_{j}$ to $s_{j+1}$ in $G$. We now construct a $k$-partition of $P$ as follows. Consider the edge $\left(u_{j}, u_{j+1}\right)$ from the path in Equation (2). Let $a_{j} \in e_{i_{j}}$ and $b_{j} \in e_{i_{j+1}}$ be the two points witnessing the existence of edge $\left(u_{j}, u_{j+1}\right)$ in $E^{\prime}$ for any $j \in[1, k-1]$. Note that both $b_{j-1}$ and $a_{j}$ are from the same edge $e_{i_{j}}$ of $P$, and we set $c_{j}=\frac{b_{j-1}+a_{j}}{2}$ to be the mid-point of $b_{j-1} a_{j}$. Obviously, $c_{j}$ is also contained in $e_{i_{j}}$. Now simply consider the sequence of breakpoints $\left\{p_{0}, c_{1}, c_{2}, \ldots, c_{k-1}, p_{N}\right\}$ and the corresponding $k$-partition of $P$. We next prove that $\delta_{F}\left(Q_{j}, P\left[c_{j}, c_{j+1}\right]\right) \leq 2 \varepsilon$ for each $j \in[1, k-2]$. 


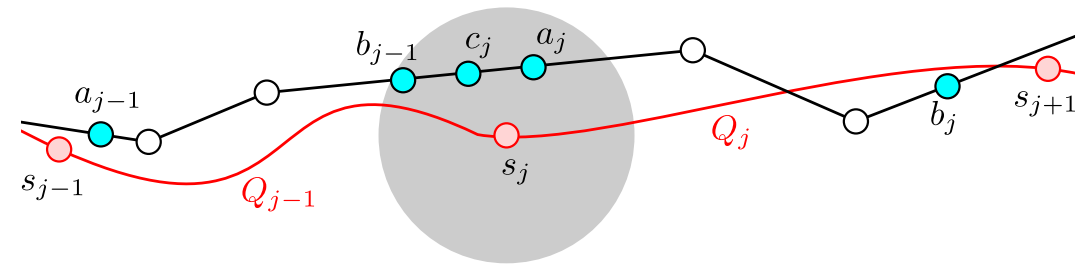

Fig. 5. Since $c_{j}$ is within the segment $b_{j-1} a_{j},\left\|s_{j}-c_{j}\right\| \leq \varepsilon$ by convexity of the $\varepsilon$-ball around $s_{j}$ (shaded region).

Indeed, by construction, we know the following:

$$
\begin{gathered}
\delta_{F}\left(Q_{j-1}, P\left[a_{j-1}, b_{j-1}\right]\right) \leq \varepsilon \\
\delta_{F}\left(Q_{j}, P\left[a_{j}, b_{j}\right]\right) \leq \varepsilon
\end{gathered}
$$

and $a_{j}, b_{j-1} \in e=e_{i_{j}}$. Figure 5 presents an illustration. Since the start point of $Q_{j}$ and the endpoint of $Q_{j-1}$ are the same, which is $s_{j}$, we then have that $\left\|a_{j}-s_{j}\right\| \leq \varepsilon$ and $\left\|b_{j-1}-s_{j+1}\right\| \leq \varepsilon$. By the triangle inequality, $\left\|a_{j}-b_{j-1}\right\| \leq 2 \varepsilon$. Since $c_{j}$ is the mid-point of $b_{j-1} a_{j}$, we have that $\left\|a_{j}-c_{j}\right\|,\left\|c_{j}-b_{j-1}\right\| \leq \varepsilon$.

Now consider the Fréchet matching $\mathcal{F}$ between $Q_{j}$ and $P\left[a_{j}, b_{j}\right]$. If $c_{j} \leq a_{j}$ (the case illustrated in Figure 5), then we can extend this matching to a matching between $Q_{j}$ to $P\left[c_{j}, a_{j}\right]$ by simply matching all points in $P\left[c_{j}, a_{j}\right]$ to the point $s_{j} \in Q_{j}$. By convexity of the distance function, we have that $\left\|p-s_{j}\right\| \leq \varepsilon$ for any point $p$ in the segment $P\left[c_{j}, a_{j}\right]$. Hence, we obtain a Fréchet matching between $Q_{j}$ and $P\left[c_{j}, b_{j}\right]$ with error still at most $\varepsilon$.

Otherwise, if $c_{j}>a_{j}$, then let $q$ be the first point in $Q_{j}$ that is matched to $c_{j}$ under the Fréchet matching between $Q_{j}$ and $P\left[a_{j}, b_{j}\right]$. Consider the subcurve $Q_{j}\left[s_{j}, q\right]$. We construct a Fréchet matching $\mathcal{F}^{\prime}$ between $Q_{j}$ and $P\left[c_{j}, b_{j}\right]$ by keeping the matching for all points in $Q_{j}\left[q, s_{j+1}\right]$ the same as in $\mathcal{F}$, but rematching all points in $Q_{j}\left[s_{j}, q\right]$ to $c_{j}$. Note that each $q^{\prime} \in Q_{j}\left[s_{j}, q\right]$ is matched to some point $p \in P\left[a_{j}, c_{j}\right]$ under $\mathcal{F}$. Hence, $\left\|q^{\prime}-c_{j}\right\| \leq\left\|q^{\prime}-p\right\|+\left\|p-c_{j}\right\| \leq 2 \varepsilon$. Thus, the new matching $\mathcal{F}^{\prime}$ has error at most $2 \varepsilon$.

By a symmetric argument, we can further modify the Fréchet matching $\mathcal{F}^{\prime}$ between $Q_{j}$ and $P\left[c_{j}, b_{j}\right]$ to a new matching $\mathcal{F}^{\prime \prime}$ between $Q_{j}$ and $P\left[c_{j}, c_{j+1}\right]$ with error at most $2 \varepsilon$. After performing this modification for all $j$, we have $\delta_{F}\left(Q_{j}, P\left[c_{j}, c_{j+1}\right]\right) \leq 2 \varepsilon$ for all $j \in[1, k-2]$. Finally, we have that $\delta_{F}\left(Q_{1}, P\left[p_{0}, c_{1}\right]\right) \leq 2 \varepsilon$ and $\delta_{F}\left(Q_{k}, P\left[c_{k-1}, p_{N}\right]\right) \leq 2 \varepsilon$ by a similar argument. The proposition then follows.

We now describe how to remove the assumption that all breakpoints in the $G$-restricted $(k, \varepsilon)$ matching have to be graph vertices. Thus, we must explain how to allow the start point and endpoint of $Q$ to lie in the interior of graph edges.

In the preceding argument, we assumed that all breakpoints of the $k$-SP path $Q$ have to be graph vertices. In our definition of $G$-restricted $(k, \varepsilon)$-matching, the first and last breakpoints of $Q$ could be points from the interior of graph edges. To allow this, we modify the auxiliary graph $G^{\prime}=$ $\left(V^{\prime}, E^{\prime}\right)$ to also add nodes of the form $\left\langle e, e_{0}\right\rangle$ or $\left\langle e, e_{N-1}\right\rangle$ to $V^{\prime}$, where $e \in E$ is any edge in input graph $G$, and $e_{0}$ (respectively, $e_{N-1}$ ) is the first (respectively, the last) edge of the input path $P$. To check whether an edge $\left(\left\langle e, e_{0}\right\rangle,\left\langle v, e_{i}\right\rangle\right)$ is in the edge set $E^{\prime}$ of the auxiliary graph $G^{\prime}$, we perform the following.

Let $e=(s, t)$ with $s, t \in V$. For any point $x \in|e|$, the shortest path from $v$ to $x$ either passes through vertex $s$, or through vertex $t$. In fact, there exists a point $w \in|e|$ such that for any point in segment $s w$, the shortest path from $v$ to it passes through $s$, whereas for any point in $w t$, the shortest path from $v$ to it passes through $t$. We break $e$ into two segments $e_{s}=s w$ and $e_{t}$. First 
consider $e_{s}$. For any $x \in\left|e_{s}\right|$, a shortest path from $v$ to $x$ in $G$ is the concatenation of a shortest path from $v$ to $s$ and segment $s x$. We thus construct a DAG $T_{v}$ containing all shortest paths from $v$ to $s$, plus the edge $e_{s}$. We then build the free space diagram $D_{v}=T_{v} \times \bar{P}\left[p_{i+1}, p_{0}\right]$, where $\bar{P}$ represents the path $P$ with the reverse orientation. We add an edge $\left(\left\langle e, e_{0}\right\rangle,\left\langle v, e_{i}\right\rangle\right)$ to the auxiliary graph if there is a shortest path $\pi_{v \sim x}$ in $G$ from $v$ to some point $x$ in $e_{s}$ and a point $b \in e_{i}=p_{i} p_{i+1}$ such that $\delta_{F}\left(\pi_{v \sim x}, \bar{P}\left[b, p_{0}\right] \leq \varepsilon\right.$. This decision problem can be answered by checking whether any point on the grid edge $e_{s} \times p_{0} \in D_{v}$ is reachable by a monotone path from the free region of $D_{v}$ starting from any point in the grid edge $v \times e_{j} \in D_{v}$. Such reachability can be maintained by a similar dynamic programming procedure as used earlier in time $O\left(\left|D_{v}\right| N\right)=O(m N)$. Similarly, we also check whether there is a shortest path from $v$ to a point in subedge $e_{t} \subseteq e$ that is within Fréchet distance $\varepsilon$ to the subpath $\bar{P}\left[b, p_{0}\right]$ for some $b \in e_{i}=p_{i} p_{i+1}$, using the same approach. We add edge $\left(\left\langle e, e_{0}\right\rangle,\left\langle v, e_{i}\right\rangle\right)$ to the auxiliary graph if the answer is yes.

The total number of extra edges we need to check for is $O(m n N)$, and checking for the existence of each such edge takes $O(m N)$ time. Hence, we need $O\left(n m^{2} N^{2}\right)$ extra time to build the auxiliary graph.

This time complexity, however, can be improved to $O\left(n m N^{2}\right)$ by batching the testing for all edges of the form $\left(\left\langle e, e_{0}\right\rangle,\left\langle v, e_{i}\right\rangle\right)$ for a fixed $v \in V, e_{i} \in P$, but all $e \in E$. We sketch the argument here. Let $D_{v}^{\prime}$ be the shortest path DAG rooted at $v$ to all other graph nodes in $V$. Next, for each $s \in V$, consider the set of edges $E_{s}$ incident on $s$ but not in $D_{v}^{\prime}$. For each such edge $e=(s, t) \in E_{s}$, we compute the furthest point $w \in|e|$ from $s$ such that the shortest path in $G$ from $v$ to $w$ passes through graph node $s$. We then add the partial edge $w s$ to the DAG $D_{v}^{\prime}$ (note that it is possible that $w=s$ ). We do this for all edges in $E_{s}$ for all nodes $s \in V$. The resulting modified DAG is denoted by $D_{v}$. We call edges in $D_{v} \backslash D_{v}^{\prime}$ partial edges. We then run the same dynamic programming procedure to compute the reachability on all grid edges $e^{\prime} \times p_{0}$ for all partial edges $e^{\prime}$ in $O\left(\left|D_{v}\right| N\right)=O(m N)$ as before. For any partial edge $e^{\prime}$ that is a subsegment of edge $\left(s^{\prime}, t^{\prime}\right) \in E$, if any point is reachable, then we add the edge $\left(\left\langle\left(s^{\prime}, t^{\prime}\right), e_{0}\right\rangle,\left\langle v, e_{i}\right\rangle\right)$ to the auxiliary graph. Overall, we need to perform this construction $O(n N)$ times for all $v \in V$ and $e_{i} \in P$. Hence, the total construction time is $O\left(n m N^{2}\right)$. This completes the proof of Proposition 5.3.

Recall that our main result, Theorem 5.1, then follows from Propositions 5.2 and 5.3.

Remark. We conjecture that we can develop an algorithm, with approximation factors better than $(2,2)$, for example, by constructing an approximate matching directly instead of going through an intermediate $G$-restricted matching. We leave this as a direction for future research.

\section{DISCUSSION AND FUTURE WORK}

In this article, we present the first algorithms for map-matching where we restrict possible matching candidates to consist of shortest paths in the graph. This variant arises naturally given the nature of GPS data, as many routing algorithms prefer certain types of paths; shortest paths are natural in this setting, but similar algorithms could be investigated in more complex settings, such as least cost roads or shortest travel time paths.

We are able to give exact algorithms for the case where shortest paths go between vertices in the graph; however, these techniques will not generalize to give exact algorithms when the shortest paths begin or end in the middle of an edge. Even our approximation for this setting does not allow the two consecutive shortest paths to reverse in the middle of an edge. Further investigation and extensions of these algorithms, as well as improved running time, are perhaps the next natural area of investigation in this work. 


\section{REFERENCES}

[1] Mahmuda Ahmed and Carola Wenk. 2012. Constructing street networks from GPS trajectories. In Proceedings of the 20th Annual European Symposium on Algorithms. 60-71.

[2] Helmut Alt, Alon Efrat, Günter Rote, and Carola Wenk. 2003. Matching planar maps. fournal of Algorithms 49 (2003), $262-283$.

[3] Helmut Alt and Michael Godau. 1995. Computing the Fréchet distance between two polygonal curves. International Journal of Computational Geometry \& Applications 5 (1995), 75-91.

[4] Theo A. Arentze and Harry J. P. Timmermans. 2004. A learning-based transportation oriented simulation system. Transportation Research Part B: Methodological 38, 7 (2004), 613-633. DOI : https://doi.org/10.1016/j.trb.2002.10.001

[5] Michael Balmer, Kay Axhausen, and Kai Nagel. 2006. Agent-based demand-modeling framework for large-scale microsimulations. Transportation Research Record: Journal of the Transportation Research Board 1985 (2006), 125-134. DOI : https://doi.org/10.3141/1985-14

[6] Sotiris Brakatsoulas, Dieter Pfoser, Randall Salas, and Carola Wenk. 2005. On map-matching vehicle tracking data. In Proceedings of the 31st Conference on Very Large Data Bases (VLDB'05). 853-864.

[7] Erin W. Chambers, Brittany Terese Fasy, Yusu Wang, and Carola Wenk. 2018. Map-matching using shortest paths. In Proceedings of the 3rd International Workshop on Interactive and Spatial Computing. 44-51.

[8] Ron Dalumpines and Darren M. Scott. 2017. Determinants of route choice behavior: A comparison of shop versus work trips using the potential path area-gateway (PPAG) algorithm and path-size logit. Fournal of Transport Geography 59 (2017), 59-68. DOI : https://doi.org/10.1016/j.jtrangeo.2017.01.003

[9] Maurice Fréchet. 1906. Sur quelques points du calcul fonctionnel. Rendiconti del Circolo Matematico di Palermo 22, 1 (1906), 1-74.

[10] Amin Gheibi, Anil Maheshwari, and Jörg-Rüdiger Sack. 2016. Minimizing walking length in map matching. In Proceedings of the International Conference on Topics in Theoretical Computer Science. 105-120.

[11] Mithilesh Jha, Samer Madanat, and Srinivas Peeta. 1998. Perception updating and day-to-day travel choice dynamics in traffic networks with information provision. Transportation Research Part C: Emerging Technologies 6, 3 (1998), 189-212. DOI : https://doi.org/10.1016/S0968-090X(98)00015-1

[12] Antonio Lima, Rade Stanojevic, Dina Papagiannaki, Pablo Rodriguez, and Marta C. González. 2016. Understanding individual routing behaviour. Fournal of the Royal Society Interface 13, 16 (2016), 20160021. DOI: https://doi.org/10. 1098/rsif.2016.0021

[13] Yin Lou, Chengyang Zhang, Yu Zheng, Xing Xie, Wei Wang, and Yan Huang. 2009. Map-matching for low-samplingrate GPS trajectories. In Proceedings of the 17th ACM SIGSPATIAL International Conference on Advances in Geographic Information Systems. 352-361.

[14] E. J. Manley, J. D. Addison, and T. Cheng. 2015. Shortest path or anchor-based route choice: A large-scale empirical analysis of minicab routing in London. Journal of Transport Geography 43 (2015), 123-139. DOI : https://doi.org/10. 1016/j.jtrangeo.2015.01.006

[15] Paul Newson and John Krumm. 2009. Hidden Markov map matching through noise and sparseness. In Proceedings of the 17th ACM SIGSPATIAL International Conference on Advances in Geographic Information Systems. 336-343.

[16] Carlo Prato and Shlomo Bekhor. 2006. Applying branch-and-bound technique to route choice set generation. Transportation Research Record: Journal of the Transportation Research Board 1985 (2006), 19-28. DOI: https://doi.org/10. 3141/1985-03

[17] Piotr Szwed and Kamil Pekala. 2014. An incremental map-matching algorithm based on hidden Markov model. In Artificial Intelligence and Soft Computing. Lecture Notes in Computer Science, Vol. 8468. Springer, 579-590.

[18] Muhammad Reaz Uddin, Chinya Ravishankar, and Vassilis J. Tsotras. 2011. A system for discovering regions of interest from trajectory data. In Advances in Spatial and Temporal Databases. Lecture Notes in Computer Science, Vol. 6849. Springer, 481-485.

[19] Jing Yuan, Yu Zheng, Chengyang Zhang, Wenlei Xie, Xing Xie, and Yan Huang. 2010. T-Drive: Driving directions based on taxi trajectories. In Proceedings of the 18th ACM SIGSPATIAL International Conference on Advances in Geographic Information Systems. 99-108.

[20] Lei Zhang, David M. Levinson, and Shanjiang Zhu. 2008. Agent-based model of price competition, capacity choice, and product differentiation on congested networks. Journal of Transport Economics and Policy 42, 3 (2008), 435-461.

[21] Shanjiang Zhu and David Levinson. 2015. Do people use the shortest path? An empirical test of Wardrop's first principle. PLoS ONE 10 (2015), 1-18. DOI : https://doi.org/10.1371/journal.pone.0134322

Received December 2017; revised January 2019; accepted September 2019 